\title{
FUTURE TRENDS IN DISTRIBUTED SIMULATION AND DISTRIBUTED VIRTUAL ENVIRONMENTS: RESULTS OF A PEER STUDY
}

\author{
Steffen Strassburger \\ School of Economic Sciences \\ Ilmenau University of Technology \\ Helmholtzplatz 3 \\ 98693 Ilmenau, GERMANY
}

\author{
Thomas Schulze \\ School of Computer Science \\ University of Magdeburg \\ Universitätsplatz 2 \\ 39106 Magdeburg, GERMANY
}

\author{
Richard Fujimoto \\ Computational Science \& Engineering \\ Georgia Institute of Technology \\ 266 Ferst Drive \\ Atlanta, G.A. 30332-0765, U.S.A.
}

\begin{abstract}
This paper reports main results of a peer study on future trends in distributed simulation and distributed virtual environments (Strassburger et al. 2008). The peer study was based on the opinions of more than 60 experts which were collected by means of a survey and personal interviews.

The survey collected opinions concerning the current state-of-the-art, relevance, and research challenges that must be addressed to advance and strengthen these technologies to a level where they are ready to be applied in day-to-day business in industry.

Most important result of this study is the observation that as research areas, both distributed simulation and distributed virtual environments are attributed a high future practical relevance and a high economic potential. At the same time the study shows that the current adoption of these technologies in the industrial sector is rather low. The study analyses reasons for this observation and identifies open research challenges.
\end{abstract}

\section{INTRODUCTION}

Distributed simulation (DS) is a technology that enables a simulation program to be executed on distributed computer systems (Fujimoto 2000). There can be many reasons and objectives for using distributed simulation. They include the desire to obtain speed-up (i.e. a reduction in the execution time of the simulation) as well as the need to couple heterogeneous and possibly geographically distributed simulation components.

Distributed virtual environments (DVEs), on the other hand, are typically defined as distributed simulations targeted at creating computer-generated virtual worlds into which users at geographically distinct locations can be embedded (Fujimoto 2000). Human interaction with DVEs and the rendering of realistic 3D visualizations typically impose real-time requirements on the execution of DVEs.

\section{SURVEY SETUP}

The survey was conducted in the period from September 15, 2007 until October 15, 2007. The survey was designed to be distributed and completed in electronic form. The survey was mainly targeted towards experts in the fields of DS/DVE. Invitations to complete the survey were therefore distributed through relevant conference distribution lists such as the Winter Simulation Conference (WSC), the Principles of Advanced and Distributed Simulation Conference (PADS), the IEEE International Symposium on Distributed Simulation and Real-Time Applications (DSRT), and the Annual Conference of the German Simulation Society (ASIM).

Furthermore the survey invitation was posted on the homepage of the Simulation Interoperability Standards Organization (SISO) and distributed to its members. It was also distributed to members of the SimSummit organization whose membership includes government, industry, and academic organizations concerned with Modeling and Simulation, especially for defense applications.

The survey questionnaire was completed by 61 individuals. The majority of the respondents $(67 \%)$ classified themselves as working in research organizations. $20 \%$ of the respondents were from industry and $10 \%$ from the defense industry. As the main intention of this study was to focus on the research aspects of the DS/DVE fields the distribution of participants agrees with our objective.

The participants were also asked to classify themselves with regard to their relationship to distributed simulation and distributed virtual environments. The responses show that $92 \%$ of the participants are directly involved with these topics, either as researcher/developer (79\%) or as practitioners $(13 \%)$.

The answers of the participants can thus be expected to give substantiated statements towards the state-of-the-art of the research in the fields as well as towards open research questions. 


\section{SURVEY RESULTS}

The survey was divided into two main parts. Part 1 was intended to address the relevance of DS/DVE technologies today and in the future. Part 2 was designed to assess open research challenges and trends in the fields under investigation. The major results of both parts are reported in the next sections

\subsection{Future Relevance of DS/DVE Technologies}

The first set of questions queried the future relevance of DS/DVE technologies to companies and other organizations. The survey suggested examples of potential areas where DS/DVE technologies could improve processes and asked the experts to rate them. The results are shown in Figure 1. Please note that all questions that asked for a rating or classification operated on a scale from 0 (none) to 5 (very high).

The diagram shows that from the suggested choices applications 2, 3 and 4 are attributed the highest relevance. The highest rated application (no. 4) concerns the application of distributed simulation to integrate heterogeneous resources. The second highest is no. 3 that involves the application of distributed simulation to join computer resources for complex distributed simulations. Both types of scenarios can be anticipated to play an important role in companies that have become sufficiently complex that they require the integration of simulation applications spanning several geographically distinct locations.

Application no. 2 involves distributed training sessions and was also assigned a high future relevance. This supports the claim that distributed virtual training sessions are a straight-forward means of joining geographically distrib-
These three applications are followed by application 5 ("Distributed Design Reviews") which still receives a ranking of 3.5 indicating that many participants still consider this a relevant topic for the future.

The usage of DVEs as a replacement of video conferencing technologies is not considered as being highly relevant for the future, however, DVEs are attributed some relevance as an enhancement of video conferencing technologies. The future could therefore bring about some mixed form of DVEs and video conferencing, e.g., by combining the traditional video feed with some interactive $3 \mathrm{D}$ view of the items to be discussed, or by integrating the video feed into the virtual world.

Participants were asked to identify additional relevant DS/DVE applications. Such applications are considered to be in the areas of production planning and control and in the area of product development. Several participants argued that both areas require the joining of planning and development processes of suppliers and OEMs. Distributed simulation is one technology which can help to overcome certain problems that arise in such situations. In the distributed planning of manufacturing lines, it can, for instance, help to join factory models developed in different simulators. In the product development phase, distributed simulation offers the potential to provide a means for protecting the intellectual property rights of different supplier companies, in so far as they can join their component models as black box models into a distributed simulation of the entire product without having to reveal knowledge about the internals of their component.

\subsection{Current Adoption}

The next question attempted to identify the current rele-

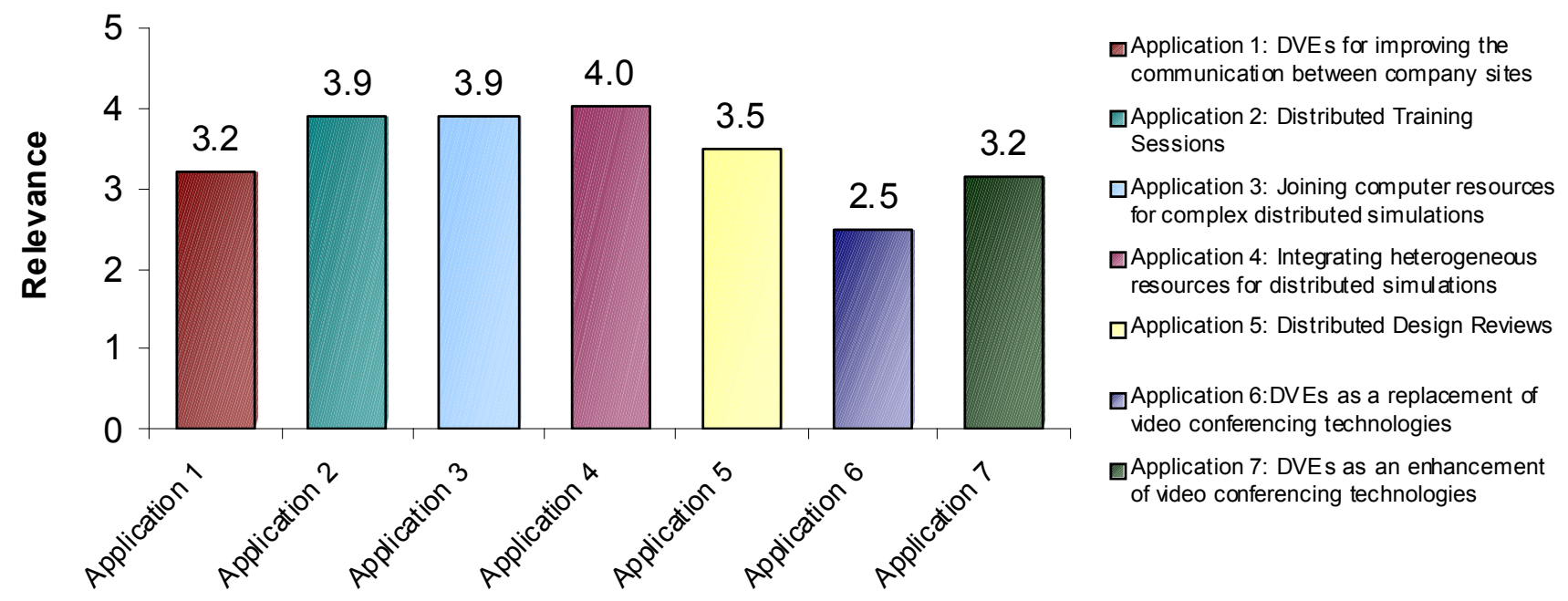

Figure 1: Potential Applications for Process Improvements

uted experts to conduct training or to provide remote assistance. vance of DS/DVE technologies. Individuals were queried for their opinion concerning the current adoption (i.e. the 
practical application) of these technologies in industry and defense. As a majority of the respondents work in research institutions, their answers can, of course, only constitute a somewhat subjective judgment of practical adoption. Still the responses provide an indication of the degree to which industry and defense differ in their usage of DS/DVE technologies.

Figure 2 shows the mean value of the responses concerning DS and DVE technology adoption in each sector (industry/defense), respectively. As expected it can be seen that the military domain already makes extensive use (between medium and high) of distributed simulation and distributed virtual environments. Military training and acquisition are typical applications that rely extensively on DS/DVE-technologies. Also, standards such as HLA and DIS originate in the defense community.

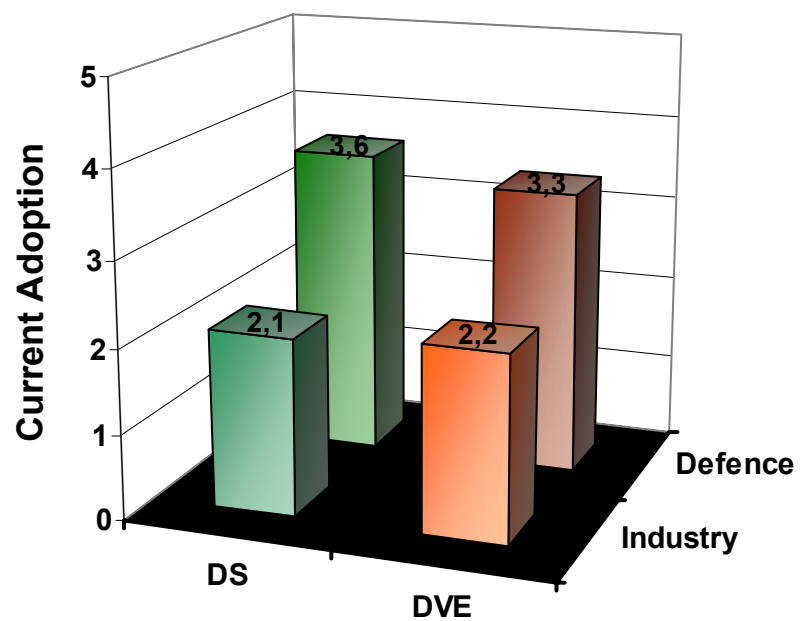

Figure 2: Current Adoption of DS/DVE-Technologies in Industry and Defense

Even though there is already strong adoption of DS/DVE technologies in the defense community, it is interesting that the degree of adoption in military applications is not rated between "very high" or "high". This may indicate the existence of technological barriers (thus indicating more research is needed), as well as organizational issues that prevent more widespread usage. This is supported by some respondents who indicated that HLA which should constitute the leading edge of standards in the military DS/DVE domain- is said to have suffered from some degree of fragmentation in the US Department of Defense, where HLA was originally created. This has resulting in the creation of additional standards (e.g., TENA). It is certainly true that even in defense today use of DS/DVE technologies has not yet reached its fullest potential.

The low usage of DS/DVE in industry, combined with the answers from the questions towards its future use suggest that industrial usage may be limited by a lack of good technical solutions and the need for more basic research in this area. It may, for instance, indicate that existing solutions are focused on the needs of the defense community and may not sufficiently take into account requirements in industry.

Some participants also indicated that DS/DVE technologies are not widely recogized in industry. This is not only due to technical issues that may prevent its usage, but also acceptance and appreciation. Concerns may arise with respect to cost and/or the invasive and disruptive character of DVEs. These problems must be overcome, along with the issue of establishing clear business cases to cover the return on investment (ROI).

This is consistent with comments on needed improvements in technical areas such as the need for seamless integration of DS/DVE with existing industrial IT infrastructures and their applications and processes.

\subsection{Economic Potential}

Respondents were also asked to estimate the economical potential of DS/DVE technologies. Although the answers cannot provide a quantitative assessment they can provide a qualitative assessment of potential economic potential.

The average value for the estimate of the economic potential is 3.7. This is a relatively high rating (4 designates "high"). This confirms that respondents believe that DS/DVE technologies do have a significant economical potential.

Respondents where also asked to identify areas where they expect the highest economic potential. Many participants indicated the defense sector as the one with the highest economic potential. Applications here include mission training and rehearsal, decision support, and technology acquisition. Indeed, the defense sector is already aligned with these technologies and the military constitutes the largest user of this technology in the simulation marketplace. In addition to defense, many participants identified the Manufacturing Sector as well as Product Development as areas with high economic potential.

Furthermore the entire area of Distance Learning Technologies (which includes advanced distributed training) is mentioned by many participants as having high economic potential.

Several participants also identified new emerging markets with an increasing economic potential. These markets include the areas of Emergency and Security Management, Homeland Security, and Global Environmental Problems.

Further responses include the gaming industry and consumer applications as well as product marketing.

A few participants also argued that economic potential is limited because of the expense related to using these technologies. This is an issue that certainly needs to be addressed, especially if the technologies are to be used by small companies that cannot afford high investments in hardware or software. 


\subsection{Influence of Changes in Business Environment}

In this question we attempted to identify societal or industrial trends which could influence DS/DVE technology usage. For example, "if the future shortage of fuel and fossil resources will lead to less travel", then "there could be a necessity for more virtual cooperation using DVEs".

The findings can be clustered into the categories "business environment" and "success stories". "Business environment" includes three main categories:

- Globalization: Economic incentives and increasing competition has lead to the formation of industrial clusters (aka globalization). This most obviously requires increased cooperation among OEMs that could establish a demand for DS/DVE technologies.

- Intellectual Property Right (IPR) Protection: With globalization and increasing cooperation among companies comes an increased need for the protection of IPR. This will increase the need for secure component-based distributed simulation in which companies can join their component models without revealing proprietary information.

- Try-before-buy: There will be an increased usage of the try-before-buy approach, i.e., selecting the right sub-components for integration in the final product from a given range of options by investigating the component's functionality and interplay with the final product using DS/DVE technologies.

The answers in the cluster "success stories" indicate quite consistently that such stories are needed to overcome psychological barriers. Also, convincing pilot applications are needed to demonstrate the positive effects of these technologies. It was further commented that technological advances must be triggered by a customer demand.

Responses to other questions highlighted technical factors which will positively influence the adoption of DS/DVE technology. Again, these answers have been clustered into two categories, "ready and robust solutions" and "technological advances."

In the cluster "ready and robust solutions" several commented that reliable standards are indispensible for a more widespread application of DS/DVE technologies by OEMs and their suppliers. Furthermore the issue of semantic interoperability must be addressed, at least within a given application domain. This requires standardized ontologies that provide out-of-the-box semantic interoperability, not only syntactic connectivity which can already be achieved today. Also, reliable tools that address these issues are needed.

In the cluster of technological advances several commented on the need for secure high bandwidth and fast network/communication technologies to enable OEMsupplier cooperation based on DS/DVE technology. Fur- thermore the adoption and emulation of game technologies were suggested to reduce entry barriers that today prevent people from applying those technologies.

An interesting comment suggested the introduction of an interim technology between traditional 2D (paper) documents and $3 \mathrm{D}$ environments in order to help people to become accustomed the new 3D technologies.

Further, many commented on the need to reduce the cost of equipment needed to adopt these technologies.

\subsection{Maturity of Existing Standards}

The survey also asked for a rating of the maturity and the practical relevance of selected standards and protocols that play an important role in the DS/DVE marketplace. Figure 3 shows the mean values derived from the survey responses.

While answers concerning the maturity of the standard can be considered quite objective, the values for the practical relevance are only significant for HLA, DIS and (to a certain degree) XMSF. This is due to the fact that the sample size varies quite significantly in the rating of the standards/protocols, i.e., if a standard was not known to a participant it did not receive a rating. HLA and DIS are known and have been rated by more than $80 \%$ of the participants. XMSF was still known by approximately $50 \%$ of the participants.

VRTP, DWTP and Mu3D on the other hand were only known by a minority of the participants (VRTP: 34\%, DWTP: $21 \%$, Mu3D: 18\%). Therefore the statements concerning the practical relevance of these protocols are questionable. Because they are known by only a minority of the participants, one could question if the rating of their practical relevance is meaningful.

The main conclusions which can be drawn from the results shown in Figure 3 are that HLA and DIS are the leading standards in the DS/DVE sector and that both already have a rather high level of maturity. On the other hand, their practical relevance is rated between medium and high (3.5 and 3.4 respectively), a value which is relatively high, but might be expected to be even higher considering that both standards have been available for more than 10 (HLA) or 15 years (DIS).

The other standards, esp. VRTP, DWTP and Mu3D are attributed a rather low maturity and only have a minor practical relevance (as explained above).

For XMSF the evaluation of the rating and the comments indicate that while the concepts of XMSF did have quite a good recognition in the community, XMSF itself does not seem to be widely supported as an on-going activity.

The participants were also asked to comment on the specific weaknesses which they would attribute to the standards and protocols. The following lists the weaknesses attributed to HLA and DIS. 


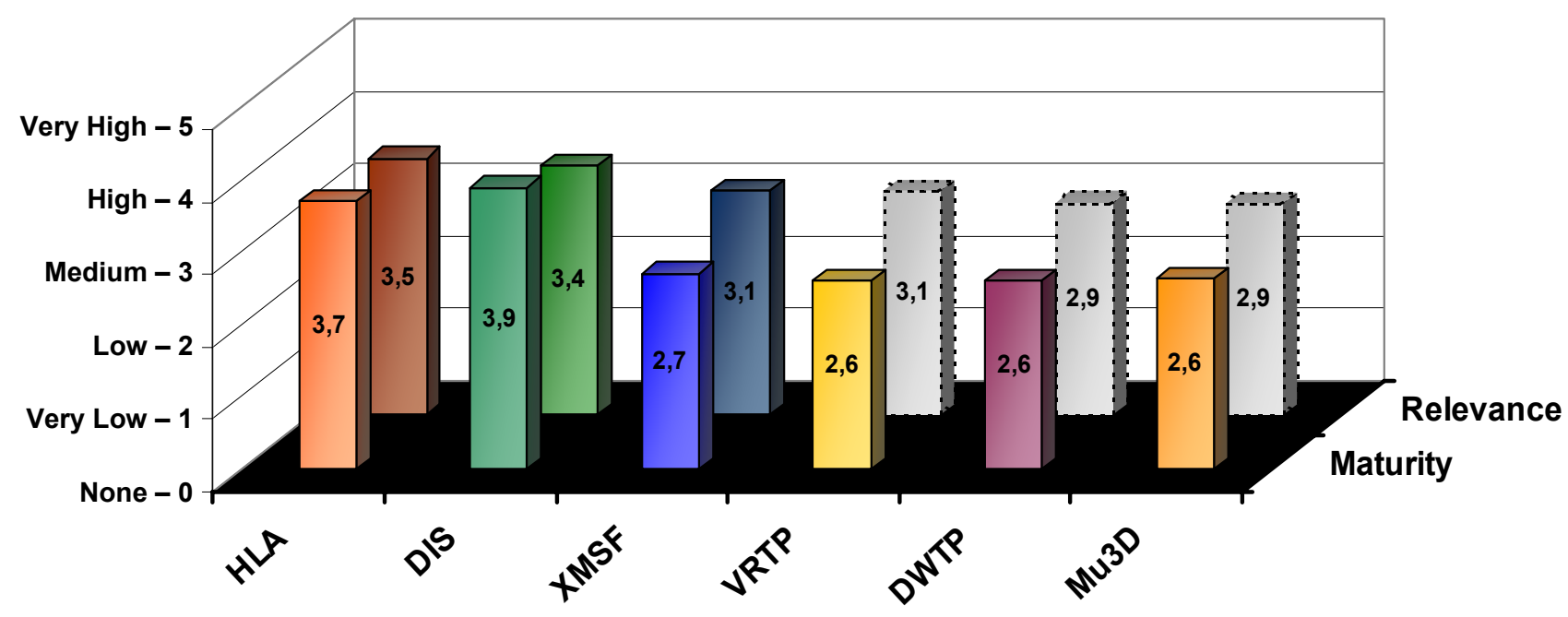

Figure 3: Maturity and Relevance of DS/DVE Standards and Protocols

Weaknesses of HLA:

- No load-balancing as part of the standard

- Poor Scalability

- Too much reliance on peer-to-peer structures, large DVEs may be better for client-server architectures supporting multiple servers

- Covers only syntactic (not semantic) interoperability

- Standard is too "heavy", i.e. very complex, difficult to learn and thus time consuming to adopt and use

Weaknesses of DIS:

- PDU broadcast concept allows no interest management (publish/subscribe) and no load balancing

- $\quad$ Limited conceptual versatility (i.e. only applicable to real-time simulations)

- Restriction to a single domain (military training simulations)

- Limitations of the standard lead to proprietary modifications and custom implementations that do not allow re-use outside the original application

In conclusion, the most critical issue concerning DIS is its limitation towards a certain niche of the simulation market (real-time, mostly military training applications) and its broadcast mechanism. HLA as the current state-of- the-art standard is attributed to at least the perception of limited scalability and the fact that it only addresses the syntactic, but not semantic interoperability issues.

\subsection{Maturity of Base Technologies}

Another question attempted to identify the most promising research areas concerning base technologies for DS/DVE applications. To do so the survey participants were asked to rate the current maturity of certain base technologies and their significance for advancing the fields of DS/DVE. The results are displayed in Figure 4.

To identify interesting and promising research areas, one should examine the base technologies that currently have the lowest degree of maturity, but still have a high rating towards its significance in advancing the DS/DVE field.

In this sense, this indicates that distributed simulation middleware, human-computer-interfaces, and the semantic web (as a placeholder for approaches supporting semantic interoperability) are the fields rated as the most promising areas of research.

On the other hand, as the maturity rating of graphics hardware is already high, no breakthroughs in this area are expected unless some completely new paradigm develops (e.g. "no triangles needed any more"). A similar observation applies to the area of network technologies.

Besides these statistics it is also interesting to examine the comments provided by the respondents concerning the improvements needed within each of the base technologies. The most important are listed below. 
Network Technologies

- Lower latency and higher bandwidth

- Robustness and fault tolerance

- Security

- Quality of Service (QoS) specifications

Distributed Simulation Middleware

- Plug-and-Play capability

- Standardization (also: Interoperability between different standards)

- Semantic connectivity

- Ubiquity (accessible anywhere with any device)

Human-Computer-Interfaces

- Multimodal interfaces, including haptics, without data gloves

- More immersive environments

- Enhanced reality instead of virtual reality required

- Usability improvements

- Human-centered interfaces

Semantic Web

- More mature ways than current ontologies for defining semantics (ontologies cannot be the only answer)

- Standardization of terms of reference for certain domains

- Ways to transform current know-how stored in the WWW into a semantic web
Graphics Hardware

- Better physics integration

- Promotion of standardization

High Performance Computing

- Involvement of all heterogeneous nodes into a universal grid

- Demonstration of application potential to broader community

- Tools to use high performance computing in engineering software environments

\section{7 "Killer" Applications}

The study also attempted to identify potential "breakthrough" applications that could drive the DS/DVE adoption forward. Responses were limited to non-military applications, since the military domain already has convincing use cases for those technologies.

The answers have been clustered into the following categories:

- Decision support systems for homeland security/catastrophes/crisis situations

Many responses fall into this category. The envisioned decision support systems are complex and networked IT systems that provide the operator/decision maker with simulation support for crisis simulations. Possible applications include simulation of the effects of a crisis (e.g. on

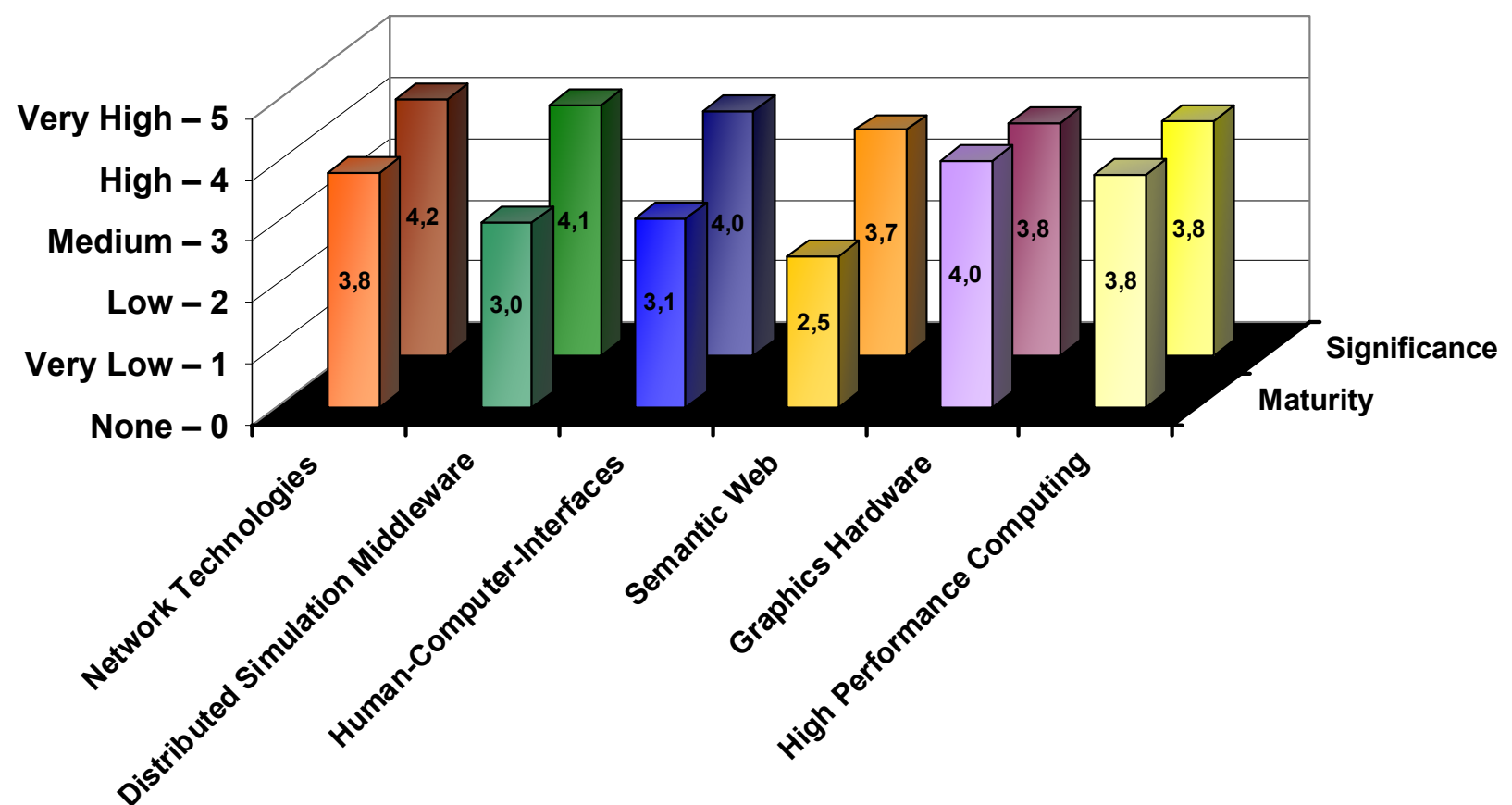

Figure 4: Maturity and Significance of Base Technologies 
complex critical infrastructures) and potential counter measures, e.g., for rescue operations.

As this simulation capability is required on demand this creates the need for powerful simulation capabilities (possibly on massively parallel architectures) and the integration of heterogeneous components for the simulation (life data feeds, data bases, geographical information systems, and multiple command and control centers).

Another envisioned element of such decision support systems concerns virtual training (and possibly remote assistance) for rescue teams.

- Virtual Training Applications in general

As virtual training applications are quite successful applications for DVEs in the military domain, one might anticipate applications of this technology in industry. Nominations in this category suggest virtual training in truly geographically and internationally distributed contexts (e.g. for the International Space Station, ISS) and training applications that combine one or multiple users with real and simulated equipment.

\section{- $\quad$ Space exploration}

Nominations in this category suggest the application of DS/DVE as a Command and Control tool for remote operations, especially in space exploration missions. With these technologies it becomes possible to send humans where they cannot go physically.

\section{- Virtual Meetings}

As this is one of the most obvious DVE applications several nominated this as a killer application. Such virtual meetings are suggested for technological development and design teams, for project progress meetings, as well as for social interaction and entertainment.

\section{- Industrial Supply Chain Simulation}

The necessity for distributed simulation applications for global supply chain simulation and optimization is motivated by the need for intellectual property protection. As the individual members of a supply chain typically do not want to reveal knowledge about their internal processes to the others a traditional (monolithic) supply chain simulation model cannot be built. DS provides a means for each participant in the supply chain to submit their supply chain node model as a black box into the overall distributed supply chain simulation.

\subsection{Research Challenges and Trends}

The survey further tried to identify the research challenges which, if solved, will advance the fields of DS/DVE significantly. The most compelling problems that were identified are briefly discussed below.

1. Solving the intrinsic conflict between desire for high interactivity / response times and the need for maintaining consistency in DVEs

A well known problem of DVEs is the need to maintain consistency among all users. Consider, for instance, a situation, where a user tries to grab a virtual part in his local representation of the DVE. To make sure that he can in fact grab it and that no other user can do so at the same time, the DVE would need to implement some locking mechanism that needs to synchronize with all participants before the user can actually grab the part. This is in conflict with providing a fast response time.

2. Easy to use synchronization algorithms which idealistically solve the "zero lookahead problem"

Efficient synchronization algorithms are the key factor for any parallel or distributed simulation. The topic of synchronization algorithms has been studied for a number of years. Several protocols (conservative, optimistic, hybrid) have been developed in the past. However, protocols that provide both ease of use and implementation and efficient execution, especially for simulations that have very low "lookahead" due to closely interacting components have not yet been found.

\section{True plug-and-play capability}

As yet no standard has enabled simulation packages to be coupled in a true plug-and-play fashion. What is needed is a technical approach and standards to couple the distributed models and gain acceptance for this standard by industry (simulation tool vendors as well as end-users). Only this can lay the basis for effortless integration of independently developed simulators and simulation components. This also requires approaches for interoperability across multiple levels of resolution, i.e., models that operate on different levels of detail.

4. Automatic or semi-automatic semantic interoperability between domains (ontologies, standard reference models, metamodels)

This challenge is a sub-challenge of the previous one. In order to achieve plug-and-play interoperability between simulations, some methodology for gaining semantic interoperability between domains (or even within a domain) 
must be established. So far, standards like HLA have mainly addressed the syntactic interoperability between simulation systems.

In addition to these challenges, participants were also asked to indentify trends that will influence the DS/DVE fields. Responses include the increasing popularity of personal computing devices, the existence of ambient networks as well as the expectation of instant and easy communications. While this may lead to new forms of accessing and using these technologies, this also imposes new research requirements, as solutions and algorithms for the special requirements of this form of usage need to be developed. As an example, algorithms for participating in DVEs under the special requirements and conditions of a mobile phone (low power consumption profile, unreliable communication, limited display size, easy interaction mechanisms) are required.

\section{SUMMARY}

The peer study summarized in this paper has investigated future trends in the fields of distributed simulation and distributed virtual environments. The survey results show that both DS and DVE are characterized as having a high practical relevance for improving both the processes within organizations and the overall product life cycle of future products. The greatest practical relevance is considered to be in the areas of joining and integrating (possibly heterogeneous) computer resources for conducting complex distributed simulations as well as in the execution of distributed training sessions.

Important applications are also considered to be in the areas of production planning and control, product development, and the general integration of geographically distributed computing resources for stakeholders. DS/DVE technologies are also attributed to having a considerable economic potential.

The survey indicates that the current adoption of DS/DVE technologies in industry today is limited. While the defense sector already makes better usage of those technologies, a lower industrial usage may be attributed to the need to articulate a clear business case for the adoption of the technologies. Although there is a high economical potential and a high practical relevance of certain applications there are a limited number of success stories and publications articulating the return on investment in using these technologies.

On the other hand, technological immaturities in these technologies exist and have been reported in this study, preventing wide-spread usage of both technologies. These immaturities help explain the different levels of usage of DS/DVE in industry vs. defense: Because the existing solutions and standards are focused on the needs of the defense community they may not take into account commercial requirements to a sufficient degree. This is in large part confirmed by the study, e.g., by revealing that there are no plug-and-play capable standards for industrial usage of DS/DVE or that there is no automatic interoperability between domains because of the lack of semantic interoperability.

As this is the case, any industrial usage must overcome the need to perform a costly integration of the needed tools, a fact that prevents a more widespread usage even if the practical application (e.g., a distributed design review) exists. That there still are significant technical and conceptual weaknesses is also confirmed by the assessment of the study that DS/DVE technologies are generally considered to still be "in the process of maturing".

The study has also revealed that there are, in fact, already some instances of DVEs which are quite successful outside the defense sector. One example of such a DVE is Second Life used for social interaction and entertainment. Their industrial usage, however, is typically limited to marketing purposes for larger companies. More serious industrial usage is prevented by conceptual and technical weaknesses.

New applications areas that could drive the DS/DVE market forward include areas such as decision support systems for homeland security and crisis management, virtual training applications, space exploration, and virtual meetings.

As for the industries and participants which will drive future innovation in these fields, it is certainly the defense industry that will have a leading role as well as the gaming industry. The gaming industry in general already has some very good proprietary solutions for implementing DVEs. However, their decision makers have little interest in revealing their solutions or in standardization efforts. On the contrary, the quality of their solutions contributes largely to the success of their products and is thus almost always considered as proprietary intellectual property.

On the other hand, this study has also shown a growing interest and need for these technologies in other industries. Especially any high-tech industry (e.g., the automotive and aeronautics industries, as well as manufacturing) will see an increasing demand for their application. This increasing demand is directly derived from the trend toward globalization. As products are often composed of parts developed and manufactured by multiple enterprises, DS and DVE technologies will make significant contributions in product development as well as in production.

Based on this growing interest standardization bodies such as the Simulation Interoperability Standards Organization through its product development groups (PDG) have begun to develop standardized solutions for enabling better DS/DVE interoperability in selected non-military applications. Examples include the Commercial Off-the-Shelf Simulation Package Interoperability (CSPI) and the Core Manufacturing Simulation Data (CSMD) PDGs. 


\section{ACKNOWLEDGEMENTS}

The authors of this study would like to express their acknowledgements to all experts who have contributed to this study, either by completing the survey questionnaire or by providing valuable discussion on the topics of this survey.

\section{REFERENCES}

Fujimoto, R. 2000. Parallel and Distributed Simulation Systems. Wiley Interscience, 2000.

Strassburger, S., Schulze, T., Fujimoto, R. 2008. Future Trends in Distributed Simulation and Distributed Virtual Environments. Peer Study Final Report. Version 1.0. Ilmenau, Magdeburg, Atlanta - January 17, 2008.

\section{AUTHOR BIOGRAPHIES}

STEFFEN STRASSBURGER is a professor at the Ilmenau University of Technology in the School of Economic Sciences. In previous positions he was working as head of the "Virtual Development" department at the Fraunhofer Institute in Magdeburg, Germany and as researcher at the DaimlerChrysler Research Center in Ulm, Germany. He holds a Ph.D. and a Master's degree in Computer Science from the University of Magdeburg, Germany. His research interests include the topics simulation and distributed simulation as well as general interoperability topics within the digital factory context. Mr. Strassburger is also the Vice Chair of SISO's COTS Simulation Package Interoperability Product Development Group. His web page can be found via <www. tuilmenau.de/fakww>.

THOMAS SCHULZE is a professor in the School of Computer Science at the Otto-von-Guericke-University, Magdeburg, Germany. He received the Ph.D. degree in civil engineering in 1979 and his habil. Degree for computer science in 1991 from the University of Magdeburg. His research interests include modeling methodology, public systems modeling, manufacturing simulation, distributed simulation with HLA and online simulation. He is an active member in the ASIM, the German organization of simulation. His web page can be found via <wwwwi.cs.uni-magdeburg.de>.

RICHARD FUJIMOTO is a professor and Chair of the Computational Science and Engineering Division of the College of Computing at the Georgia Institute of Technology. He received the Ph.D. and M.S. degrees from the University of California (Berkeley) in 1980 and 1983. Among his activities he was the technical lead concerning time management issues for the DoD High Level Architecture (HLA) effort, and has also been chair of the steering committee for the Workshop on Parallel and Distributed Simulation, (PADS) since 1990. His email address is <fujimoto@cc.gatech.edu>. 